\title{
PREDICTION OF PLASTIC PROPERTIES OF RODS FROM INDIVIDUAL GRAIN ORIENTATION MEASUREMENTS
}

\author{
F. BARLAT, J. LIU and R.C. DICKENSON \\ Alcoa Laboratories, Alcoa Center, PA 15069, USA
}

\section{INTRODUCTION}

Two processes are used to produce $9.5 \mathrm{~mm}$ rod from 5056 aluminum alloy. The conventional process comprises D.C. casting, homogenization, extrusion, and rod rolling. A more economical process entails continuous casting of a small bar immediately followed by rod rolling. Cylindrical compression specimens were taken out of the rods obtained by the two processes. After compression, the section of the conventionally processed rod was still circular, whereas the section of the continuously cast rod was elongated in one direction. This anisotropy leads to practical problems for any subsequent axisymmetric forming process. In this work, the crystallographic textures of rods were evaluated to determine if they were responsible for the anisotropic behavior of the compression samples. Since the continuous cast process is non-axisymmetric, structural inhomogeneity is expected. Standard X-ray diffraction techniques lack the necessary spatial resolution. Therefore, individual grain orientations were measured by means of backscattered Kikuchi diffraction using a scanning electron microscope (SEM) and a computer-aided system attached to the SEM ${ }^{1}$. For each specimen, several locations were investigated and a number of orientations were measured at each location leading to an estimate of local as well as overall average textures. Computations using the

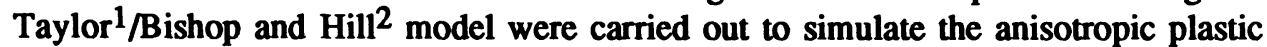
behavior of specimens subjected to uniaxial compression. Using this analysis, the theoretical aspect ratio of rods after uniaxial compression was estimated and compared to experimental results. The present method, which consists of using individual grain orientation measurements to evaluate anisotropic properties of materials, can be used in other applications where standard X-ray diffraction techniques can not be used.

\section{EXPERIMENTAL PROCEDURE AND RESULTS}

The grain orientations were measured for 5056 aluminum rod specimens fabricated by each of the two rod manufacturing processes. Sections perpendicular to the rod axis were mechanically polished and electropolished. Then, they were examined in the SEM. The imaging of the grain structure was obtained by channeling contrast. Due to symmetry considerations, only one quarter of each section was examined. For the rod processed by the conventional method, well-defined grain boundaries were observed throughout the specimen, indicating that the material was completely recrystallized. The grain size was of the order of $10 \mu \mathrm{m}$ and was rather uniform. The grain shape was equiaxed irrespective of the location. However, for the rod processed by continuous casting, the grain structure was not so homogeneous. In a region of the rod extending from the center to the edge in a direction denoted by $Z$, the grain boundaries, grain size 
and aspect ratio were very similar to those for the conventionally processed material. In a radial direction perpendicular to $\mathrm{Z}$ denoted as $\mathrm{Y}$, similar grain structure was observed to extend only from the rod center to a distance half the rod radius. However, further away from the center in the $\mathrm{Y}$ direction, most of the grain boundaries were not well-defined, indicating that the material was predominantly unrecrystallized. In fact, the unrecrystallized region corresponds to the deformation zone of the last rolling stand. In this region, the volume fraction of recrystallized grains was estimated to be $10 \%$, although no formal quantitative analysis was performed. Figs. 1 and 2 are schematic illustrations of the recrystallized (REX) and unrecrystallized (URX) areas of the 5056 rods processed by conventional or continuous casting, respectively. The two distinct regions were separated by a very narrow transition domain, so that it was easy to map the crosssections of the two conditions.

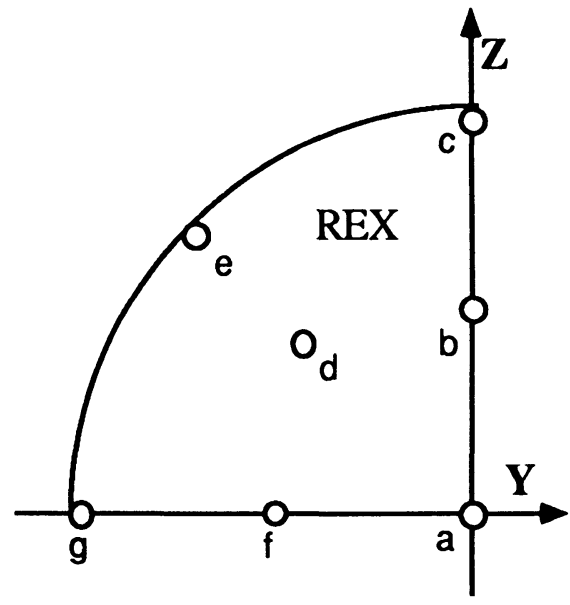

Figure 1. Schematic quarter section of 5056 rod processed by conventional casting.

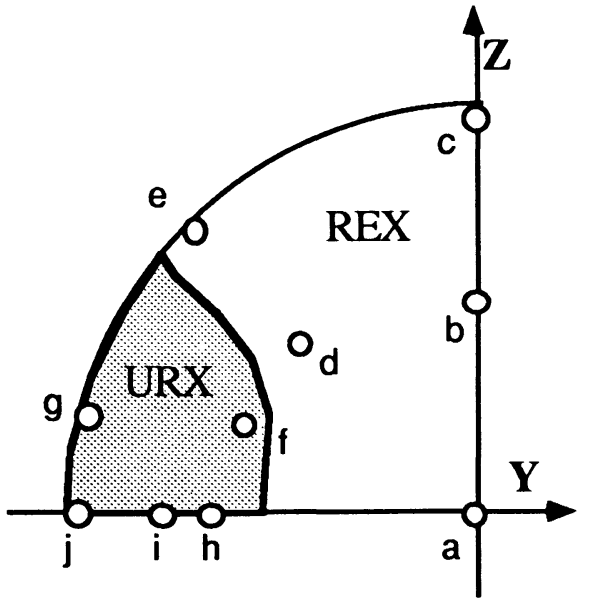

Figure 2. Schematic quarter section of 5056 rod processed by continuous casting.
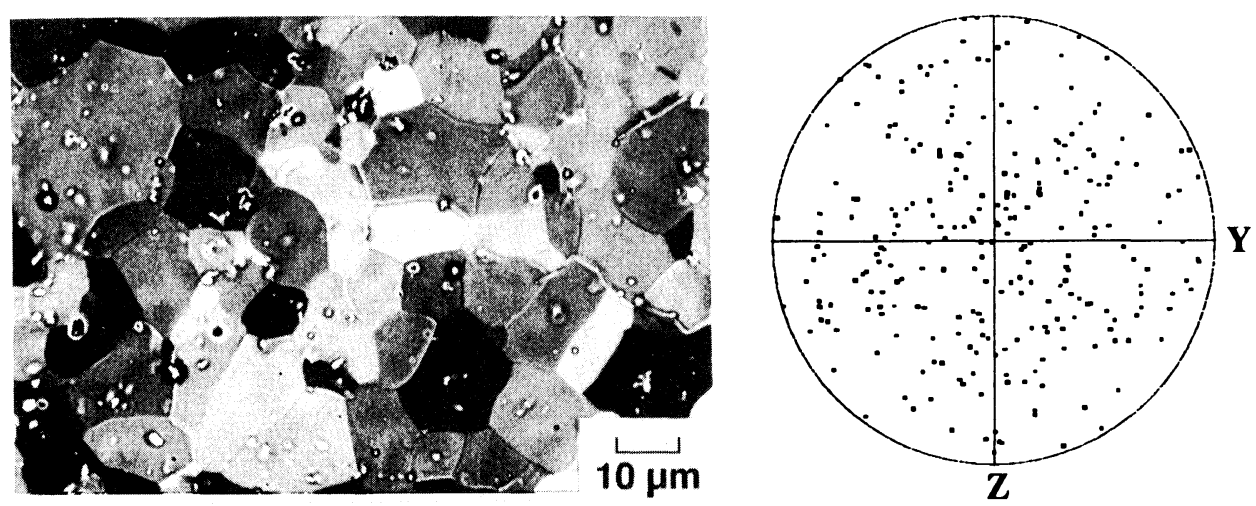

Figure 3. Typical channeling contrast micrograph of 5056 rod processed by conventional method
Figure 4. Local (111) pole figure at location (a) of 5056 rod processed by conventional casting. 
Backscattered Kikuchi patterns were used to measure individual grain orientations. A low light level TV camera captures the diffraction pattern and the processed pattern is plotted onto a computer CRT. The indexing of crystallographic planes and poles is assisted by a computer program. When the pattern has been indexed, the absolute orientation of the grain is computed. Typically, one orientation can be measured within a minute. Individual orientations were measured in different locations in the quarter section of each specimen. These locations are represented by circles in Figs. 1 and 2 and denoted by letters (a) to (g) for the conventionally processed rod and (a) to (j) for the other sample. At each location, the electron beam was successively moved to one of the nodes of an approximately square array of grids. About 64 grain orientations were measured at each location. A total of $\mathbf{4 4 1}$ grains were measured in the rod processed by conventional casting, while 337 and 330 orientations were measured in the URX and REX regions, respectively, of the continuously cast sample.

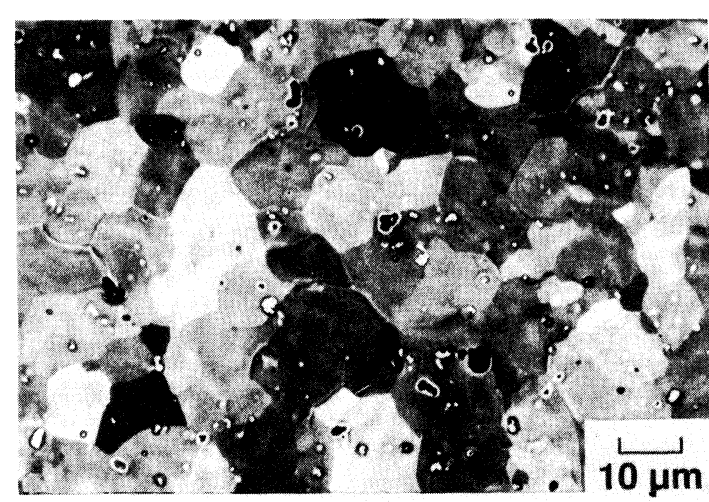

Figure 5. Typical channeling contrast micrograph of 5056 rod processed by continuous casting ( $R E X$ region).

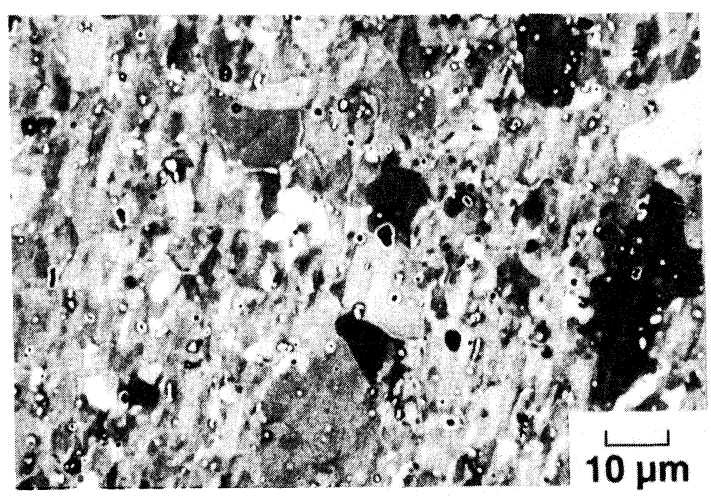

Figure 6. Typical channeling contrast micrograph of 5056 rod processed by continuous casting (URX region).

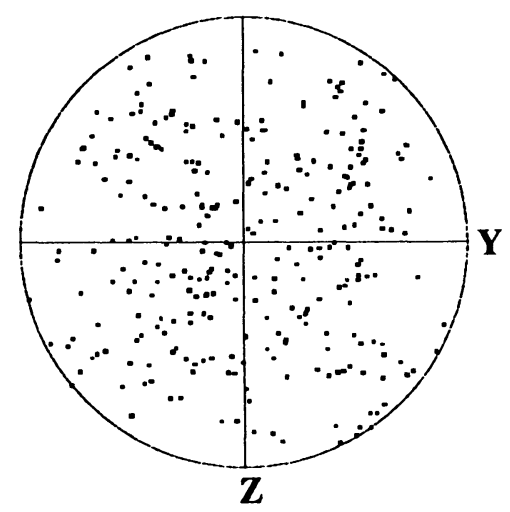

Figure 7. Local (111) pole figure at location (a) of 5056 rod processed by continuous casting (REX).

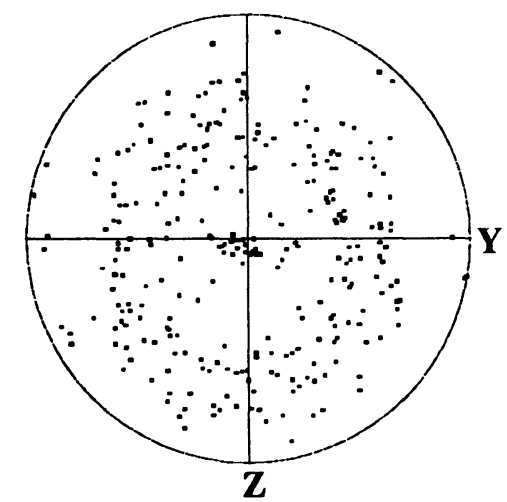

Figure 8. Local (111) pole figure at location $(j)$ of 5056 rod processed by continuous casting (URX). 
Fig. 3 is a typical channeling contrast micrograph of 5056 rod processed by the conventional method. The distance between each electron beam spot was around $40 \mu \mathrm{m}$. Since the grain size was about $10 \mu \mathrm{m}$, the measurements were independent of each other. The recrystallized grains produced sharp and easily indexable patterns. Fig. 4 is the (111) pole figure at location (a) in the middle of the rod. It shows an isotropic distribution of orientations rather than a fiber type of texture. The pole figures at the other locations in the conventionally cast rod exhibited the same trend. No particular preferred orientation was evident from the pole figures.

Figs. 5 and 6 show the grain structure of the 5056 rod processed by continuous casting in the recrystallized (REX) and unrecrystallized (URX) regions, respectively. The grain structure of the REX region (Fig. 5) is similar to the structure of the other rod (Fig.3). Grain boundaries were not so well-defined in the URX region. The subgrains here are only slightly larger than the spot size of the electron beam and the Kikuchi patterns were somewhat blurred due to the slight overlapping of several patterns. However, it was always possible to index them properly. Figs. 7 and 8 are the (111) pole figures at the center of the rod and at location (j), respectively. These figures indicate that the texture is somewhat stronger in this specimen than that in conventionally processed rod. However, at first sight no particular difference can be observed between $Y$ and $Z$ directions. In the center of the specimen, the texture seems to exhibit a mirror symmetry with respect to both axes (Fig. 7) whereas, at location (j), the texture appears to exhibit a fiber symmetry (Fig. 8).

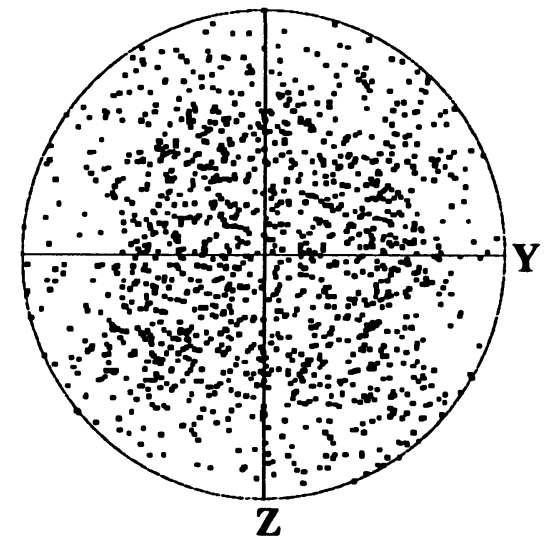

Figure 9. 111 pole figure for continuously cast rod in region $R E X$.

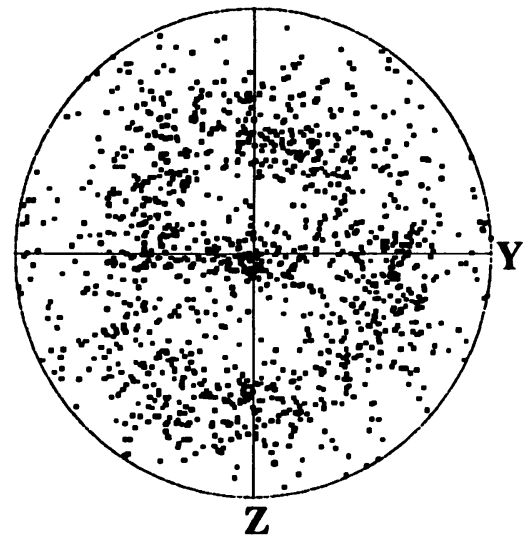

Figure 10. 111 pole figure for continuously cast rod in region URX.

For a cold rolled plate or sheet, all points in the material typically have a similar texture, and the pole figures are plotted with respect to the rolling and transverse directions. However, in the present case of rods, the texture is not uniform at all locations. During the forming process, the rod moves in successive rolling stands where the roll axis is alternately parallel to either the $\mathrm{Y}$ or the $\mathrm{Z}$ direction. Even though the rolls are curved, the rods can be considered to be orthotropic as a first approximation. This is supported by Figs. 7 and 8. As a result, all of the local pole figures for the REX and URX locations have been represented in the same $\mathrm{Y}$ and $\mathrm{Z}$ orthotropic axes, and they all resemble the pole figures shown above (Figs. 7 and 8). Because of this particular symmetry, it is reasonable to represent all the orientations corresponding to either the 
REX or the URX region in the same 111 pole figure (Figs. 9 and 10). These figures give the average texture in both areas (REX and URX). It is worthy to note that the local (111) pole figures (Figs. 7 and 8) are comparable to the global (111) pole figures (Figs. 9 and 10). So, it seems that locations (a) and (j) are representative of the average texture of the 5056 rod processed by continuous casting and that 64 grain orientations are sufficient to give a good idea of the crystallographic texture in either region of the material. However, only Fig. 10 clearly shows that directions $\mathrm{Y}$ and $\mathrm{Z}$ are not equivalent and that the rod can exhibit anisotropic behavior.

Compression tests were conducted for both 5056 rod conditions. After deformation, the radius of the rod was measured in both directions $Y$ and $Z$. The ratio $R_{y} / R_{z}$ was approximately 0.985 and 1.079 at a true strain of -1.46 and -1.19 for the rods processed by conventional and continuous casting, respectively. After compression, crystallographic texture measurements were made by conventional X-ray techniques. For both rod conditions, the resulting (111) pole figures exhibit an orthotropic though close to a fiber symmetry.

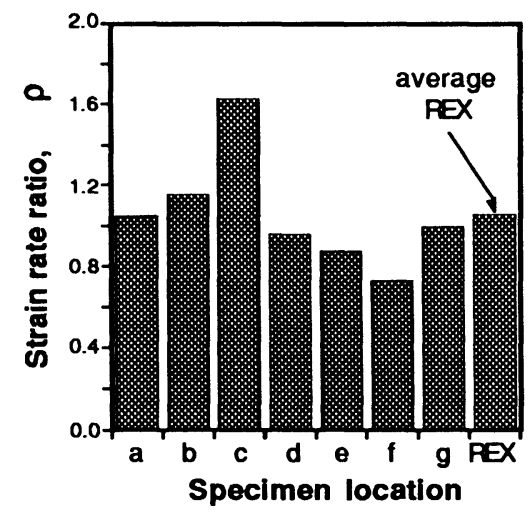

Figure 11. Initial strain rate ratio $\rho$ calculated in uniaxial compression for conventionally cast rod.

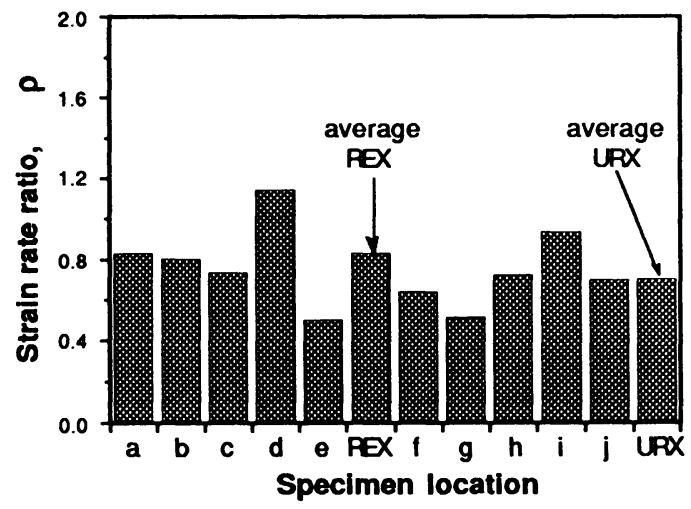

Figure 12. Initial strain rate ratio $\rho$ calculated in uniaxial compression for continuously cast rod.

\section{DISCUSSION}

The Taylor ${ }^{2} /$ Bishop and Hill ${ }^{3}$ model was used to calculate the ratio $\rho$ of the strain rates in the $\mathrm{Y}$ and $\mathrm{Z}$ directions of a rod, when an uniaxial stress is imposed in the $\mathrm{X}$ direction (rod axis):

$$
\rho=\frac{\dot{\varepsilon}_{y y}}{\dot{\varepsilon}_{z z}}
$$

This ratio was calculated for each local area of both 5056 rods, as well as in the average REX and URX regions. This data are represented in Figs. 11 and 12 for both materials. Comparison of these figures shows that parameter $\rho$ is smaller for the rod processed by continuous casting, with an average value of 0.83 and 0.70 for the REX and URX regions, respectively. This indicates that both URX and REX regions are anisotropic. It is interesting to note that $\rho$ is less than 1 in almost every location for this continuously 
cast rod, indicating that the ratio $R_{y} / R_{z}$ is larger than 1.0 . So, almost every local pole figure with 64 orientations is able to predict the correct anisotropic behavior. For the conventional material, the average value of $\rho$ is close to 1 (1.06), indicating nearly isotropic behavior.

The ratio $R_{y} / R_{z}$ can be calculated as a function of the the compressive strain $\varepsilon_{x x}$ and assuming incompressible deformation. Since the crystallographic texture and other microstructural features evolve during deformation, the ratio $\rho$ is not constant but depends on the strain, $\rho=\rho\left(\varepsilon_{x x}\right)$. Regardless of the form of this function, the ratio between the radii in the $Y$ and $Z$ directions can be given by:

$$
\frac{R_{y}}{R_{z}}=\exp \left(\int_{0}^{\varepsilon_{x x}} \frac{1-\rho}{1+\rho} d \varepsilon_{x x x}\right)
$$

The variation of $\rho$ as a function of the compressive strain could be calculated using a polycrystalline model that takes texture evolution into account. However, as a first approximation, $\rho$ can be assumed to vary linearly from its initial value $\rho_{0}$ to 1 , since after compression, the resulting crystallographic texture exhibits a fiber-like symmetry, with an expected value of $\rho=1$. Then, using Equ. 1 and the average value $\rho_{0}=1.06$ and $\rho_{0}=0.8$ for the conventionally and the continuously cast material, the calculated ratio of the two radii is 0.979 and 1.066 , respectively. This is in reasonable agreement with the experimental values, 0.985 and 1.079 , respectively.

The measurement of individual grain orientations in the SEM is a reliable method for qualitative and quantitative descriptions of crystallographic texture and anisotropy. Unlike conventional X-ray methods, it can be used for small size products, as in this work, and it can describe local features of the crystallographic texture. However, it seems that a few hundred grain orientations are necessary to capture the quantitative aspect of the problem. Figs. 11 and 12 indicate that uncertain conclusions would be made based on the measurement of 64 grains at one or two locations of a specimen. In addition to the SEM-based individual orientation technique, the TEM-based single orientation capability ${ }^{4}$ has also been recently automated. To bridge the individual and bulk orientation techniques, the TEM pole figure method $^{5}$, which has also been developed, could be used for further studies of these rods. In the future, we plan to use polycrystal models to account for the texture evolution. This will allow computation of the parameter $\rho$ as a function of compressive strain to get a better estimate of the ratio of the final radii in the directions $Y$ and $Z$, and to compare theoretical and experimental textures after compression. Moreover, it would be interesting to determine if an initially anisotropic texture eventually exhibits a fiber symmetry after compression.

\section{REFERENCES}

1. D.J. Dingley, Scanning Electron Microscopy, II, 569 (1984).

2. G.I. Taylor, J. Inst. Metals, 62,307 (1938).

3. J.W.F. Bishop and R. Hill, Phil. Mag., 42, 414 and 1298 (1951).

4. H. Weiland and R.A. Schwarzer, Experimental Techniques of Texture Analysis, (DGM, Oberursel, 1987), p. 301.

5. R.A. Schwarzer and H. Weiland, Experimental Techniques of Texture Analysis, (DGM, Oberursel, 1987), p. 287. 